\title{
Réseaux électrocinétiques et algèbre linéaire (notions fondamentales)
}

\author{
P. LAGONOTTE, Y. EICHENLAUB
}

\section{I.U.T. de Poitiers, Département GEII}

\begin{abstract}
Résumé : Cet article présente une séance de travaux pratiques transversale entre les enseignements de mathématiques et d'électricité. Le but du TP est de déterminer les valeurs de l'ensemble des résistances d'un circuit sans avoir la possibilité d'accéder individuellement à chaque élément. La résolution de ce problème de manière élégante fait appel au calcul matriciel. Après une étude complète rapide de l'aspect théorique du problème, l’article détaille tous les éléments nécessaires à la mise en œuvre pratique du TP. Des exemples logiciels et quelques notices historiques sont fournis en annexes.
\end{abstract}

Mots Clés : algèbre linéaire, matrices, systèmes maillés, électrocinétique, mesures, traitement de l’information.

Connaissances requises pour les étudiants : électricité (loi d'Ohm et lois de Kirchhoff), mathématiques (calcul matriciel). Niveau des étudiants : premier cycle universitaire.

\section{Introduction}

L'enseignement de l'algèbre linéaire peut poser des problèmes à certains de nos étudiants n'ayant pas des capacités d'abstraction importantes. En effet, les matrices leur apparaissent souvent comme des objets mathématiques abstraits, et toutes les opérations sur les matrices restent pour la plupart d'entre eux de simples mécanismes calculatoires.

Si le cours de mathématiques se doit de rester essentiellement théorique, certains étudiants peuvent se demander à juste titre : «À quoi tout cela peut-il bien servir ? ». Dans le cadre d'un cursus de formation «E.E.A.» de premier cycle universitaire, les réseaux électrocinétiques offrent des exemples simples de situations qui permettent d'utiliser de manière concrète les concepts de l'algèbre linéaire.

Notre objectif est de présenter ici une application de l'algèbre linéaire aux systèmes électriques maillés, et de montrer la puissance de cet outil mathématique pour résoudre très simplement un problème apparemment complexe comme l'analyse du fonctionnement d'un réseau électrocinétique qui comporte un grand nombre d'éléments. Sur le plan électrique, le seul outil nécessaire est la loi d’Ohm (1789-1854). L'aspect topologique du système maillé est pris en compte au niveau de la mise en équation matricielle.

Nous avons essayé de rédiger ce document pour qu'il soit facilement lisible à la fois par des mathématiciens et par des électriciens, dans le but de contribuer si possible à un certain décloisonnement de l'enseignement.

\section{Réseaux, systèmes maillés et matrices}

\subsection{La matrice des conductances [G]}

Considérons un réseau électrique maillé constitué d'éléments de conductances constantes comme présenté figure 1 , et supposons que l'on injecte en l'un des nœuds nommé $\mathbf{i}$ un courant $\mathrm{I}_{\mathrm{i}}$. Les courants à deux indices correspondent aux courants qui traversent les conductances du réseau, alors que les courants à un seul indice correspondent aux courants injectés de l'extérieur du réseau (voir vecteur [I] plus loin).

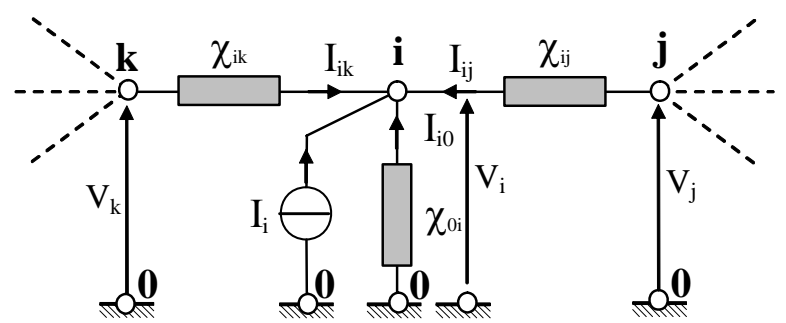

Fig. 1. Les nœuds et les branches d'un réseau maillé de conductances

La loi des nœuds (lois de Kirchhoff (1824-1887)) appliquée au nœud i s’écrit alors tout simplement :

$$
I_{i}+I_{i 0}+I_{i j}+I_{i k}=0
$$


ce qui, grâce à la loi d’Ohm, se traduit par :

$$
I_{i}=\chi_{0 i} V_{i}+\chi_{i j}\left(V_{i}-V_{j}\right)+\chi_{i k}\left(V_{i}-V_{k}\right)=\left(\chi_{0 i}+\chi_{i j}+\chi_{i k}\right) V_{i}-\chi_{i j} V_{j}-\chi_{i k} V_{k}
$$

Si l’on considère maintenant un réseau plus général, constitué également de conductances constantes, si l'on note $\chi_{\mathrm{ij}}$ (ou bien $\chi_{\mathrm{ji}}$ ) la conductance entre deux nœuds $\mathbf{i}$ et $\mathbf{j}$ quelconques, et si l'on injecte en chaque nœud $\mathbf{i} \geq 1$ un courant noté $\mathrm{I}_{\mathrm{i}}$, la loi des nœuds appliquée au nœud $\mathbf{i}$ s'écrit :

$$
I_{i}=\sum_{j \neq i} \chi_{i j}\left(V_{i}-V_{j}\right)
$$

soit encore, pour tout $\mathbf{i} \geq 1$ :

$$
I_{i}=V_{i}\left(\sum_{j \neq i} \chi_{i j}\right)-\sum_{j \neq i} V_{j} \cdot \chi_{i j}
$$

Notons [I] la matrice colonne (vecteur) des courants injectés aux nœuds (pour $\mathbf{i} \geq 1$ ), et [V] la matrice colonne (vecteur) des potentiels aux nœuds (pour $\mathbf{i} \geq 1$ ). Toutes les équations (4) correspondent exactement à l'unique équation matricielle :

$$
[\mathrm{I}]=[\mathrm{G}][\mathrm{V}]
$$

où [G] est la matrice des conductances, définie par :

- les termes diagonaux sont égaux à la somme des conductances reliées au nœud $\mathbf{i}: G_{i i}=\sum_{j} \chi_{i j}(\mathrm{j}=0$ y compris);

- les termes non diagonaux valent l’opposé de la conductance reliant le nœud $\mathbf{i}$ au nœud $\mathbf{j}$ : $G_{i j}=-\chi_{i j}$.

La matrice [G] se construit très simplement, même pour des réseaux maillés de très grande taille, en disposant de la liste des composants et de leurs deux nœuds de rattachement.

Nous pouvons noter plusieurs particularités de la matrice [G] :

- les termes diagonaux $\left(\mathrm{G}_{\mathrm{ii}}\right)$ sont tous positifs ;

- les termes non diagonaux sont tous négatifs ou nuls ;

- la matrice est symétrique $\left(\mathrm{G}_{\mathrm{ij}}=\mathrm{G}_{\mathrm{ji}}\right)$;

- pour les grands réseaux, la plupart des termes non diagonaux sont nuls: la matrice est dite "creuse". Physiquement, le bilan des courants en un nœud donné n’est rattaché qu’aux tensions des nœuds adjacents. Les interactions sont donc "locales" ;

- l'analyse de la matrice [G] permet de retrouver la topologie du réseau ;

- cette matrice s’établit de manière très naturelle à partir des éléments du réseau.

\subsection{La matrice des résistances $[R]$}

Par définition, la matrice $[\mathrm{R}]$ est simplement l'inverse de la matrice [G] (lorsque cette dernière est inversible). On a donc :

$$
[\mathrm{V}]=[\mathrm{R}][\mathrm{I}] \text {. }
$$

Cette fois-ci, la matrice [R] est une matrice pleine, car si nous injectons un courant au seul nœud i (c'est-à-dire si $\mathrm{I}_{\mathrm{j}}=0$ pour tout $\mathrm{j}$ différent de i), nous obtenons :

$$
\mathrm{V}_{\mathrm{j}}=\mathrm{R}_{\mathrm{ji}} \mathrm{I}_{\mathrm{i}} \text {. }
$$

Ainsi, $\mathrm{R}_{\mathrm{ji}}$ décrit l'élévation des potentiels dans l'ensemble du système suite à une injection de courant au seul nœud i. La matrice [R] traduit donc l'influence d'un nœud sur tous les autres ; c'est de ce point de vue une matrice de sensibilité. Physiquement, l'injection d'un courant $\mathrm{I}_{\mathrm{i}}$ au nœud $\mathbf{i}$ va engendrer des variations de tension dans l'ensemble du réseau, et pour $\mathbf{i}$ et $I_{i}$ fixés, le potentiel $V_{j}$ au nœud $\mathbf{j}$ sera proportionnel à $\mathrm{R}_{\mathrm{ji}}$.

Le terme diagonal $\mathrm{R}_{\mathrm{ii}}$ correspond à la résistance équivalente du réseau pris dans son ensemble, vu du nœud numéro $\mathbf{i}$, puisque l'on a : $\mathrm{V}_{\mathrm{i}}=\mathrm{R}_{\mathrm{ii}} \mathrm{I}_{\mathrm{i}}$. C'est l'impédance d'entrée entre le nœud $\mathbf{i}$ et le nœud de référence. Les termes non diagonaux traduisent l'influence réciproque d'un nœud sur un autre. En effet, si l'on injecte un courant $\mathrm{I}_{\mathrm{i}}$ au seul nœud $\mathbf{i}$, le rapport des tensions $\mathrm{V}_{\mathrm{i}}$ et $\mathrm{V}_{\mathrm{j}}$ est donné par l'équation : 


$$
\frac{V_{j}}{V_{i}}=\frac{R_{j i}}{R_{i i}} \text { (6) }
$$

Les rapports $R_{j i} / R_{i i}$ et $R_{i j} / R_{j j}$ correspondent donc aux atténuations des tensions entre les nœuds $\mathbf{i}$ et $\mathbf{j}$. Il faut toutefois remarquer que l'atténuation $\left(V_{j} / V_{i}\right)$ d'une perturbation appliquée en $\mathbf{i}$ et observée sur le nœud $\mathbf{j}$ n’est en général pas égale à l'atténuation de la même perturbation appliquée en j, et observée sur le nœud i !

Résumons les principales particularités de la matrice [R] :

- les coefficients de la matrice sont tous positifs ;

- la matrice [R] est symétrique : $\mathrm{R}_{\mathrm{ij}}=\mathrm{R}_{\mathrm{ji}}$;

- la matrice $[R]$ est une matrice mathématiquement pleine. Elle contient de façon intrinsèque les interactions physiques entre tous les couples de nœuds, ce qui a l'avantage de donner une vision "globale" de la diffusion en tension d'une injection de courant locale sur tous les autres nœuds du système. La matrice $[R]$ est une matrice de sensibilité ;

- cette matrice est assez difficile à établir et à interpréter à partir des éléments du réseau, mais elle est facile à établir à partir de mesures faites sur le réseau.

Comme les matrices [G] et [R] sont inverses l'une de l'autre, ces matrices contiennent intrinsèquement les mêmes informations. Mais ces informations sont présentées sous deux formes différentes : l'une locale pour la matrice [G], l'autre globale pour la matrice [R].

Remarque : Une autre interprétation des valeurs des coefficients $\mathrm{R}_{\mathrm{ij}}$ peut être donnée lorsque l'on injecte des courants $I_{i}$ et $I_{j}$ simultanément en deux nœuds $\mathbf{i}$ et $\mathbf{j}$ et en aucun autre. Il est alors d'usage en électricité de résumer le circuit par un schéma équivalent qui peut être dessiné sous deux formes : le schéma en triangle (qui est sans doute le plus naturel) et le schéma en étoile (voir figure 2)

a ) Montage en "T" ou en "Etoile"

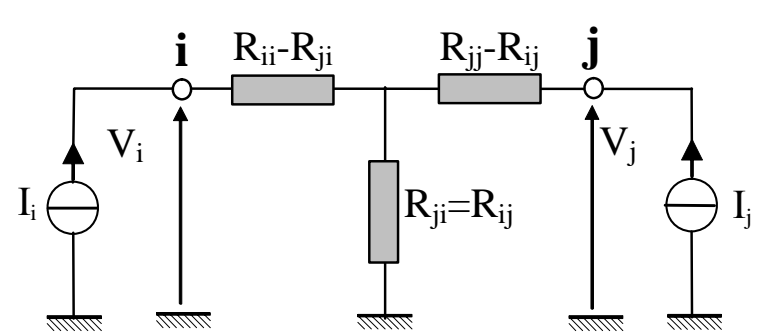

b ) Montage en " $\Pi "$ ou en "Triangle"

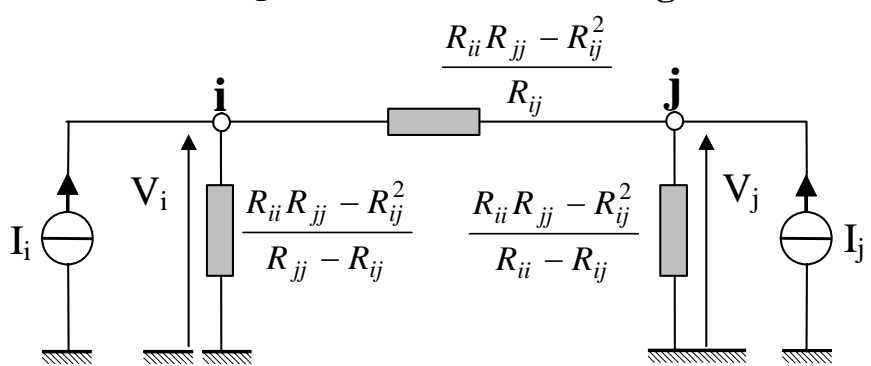

Fig. 2. L'interprétation des termes de la matrice [R] sous la forme de ponts diviseurs en « $\Pi$ » et en « $T$ »

Dans ce cas, on établit aisément que les valeurs des résistances équivalentes sont données en fonction des $\mathrm{R}_{\mathrm{ij}}$ par les formules inscrites sur la figure 3. Le schéma en étoile apporte donc une signification physique précise au coefficient $R_{i j}$ quand $\mathbf{i} \neq \mathbf{j}$.

Le passage entre les figures 2.a et 2.b peut s'effectuer par transposition « triangle-étoile » (formules d'Arthur Edwin Kennelly (1861-1939)). Remarquons aussi que seule la représentation en « $\Pi$ » ou « triangle » se généralise lorsqu’il y a plus de trois pôles.

\subsection{Matrices singulières et topologie du réseau}

\section{Nœud de référence}

L’équation du nœud de référence (numéroté 0) n’est autre que la somme des équations de tous les autres nœuds. Pour que la matrice $[G]$ ne soit pas singulière (singulière $=$ non inversible), il est donc nécessaire de ne pas tenir compte de l'équation du nœud de référence. Le fait de noter le nœud de référence 0 permet de conserver une cohérence entre le nombre de nœuds du réseau et la dimension de la matrice [G].

\section{Réseau connexe}

Un réseau est connexe s’il existe au moins un chemin pour aller d'un nœud à un autre. La figure 3 présente des exemples de topologies connexe et non connexe. Dans le cas où la topologie du réseau est 
non connexe, la matrice [G] associée au réseau est singulière. En fait, la partie du réseau (k, 0’) est électriquement isolée par rapport à la partie (i, j, 0), et la différence de potentiel entre les deux sousréseaux est non calculable.

a ) Réseau connexe : Matrice non singulière

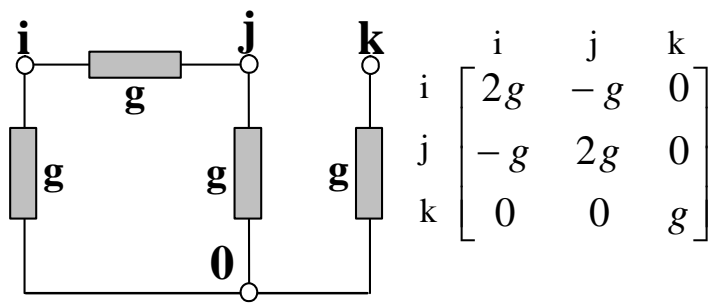

b ) Réseau non connexe : Matrice singulière

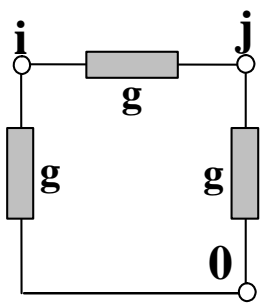

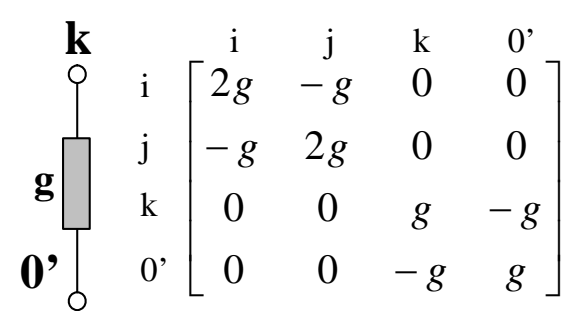

Fig. 3. Connexité des topologies et matrices singulières

\subsection{Systèmes linéaires, non réciproques et non linéaires}

Les matrices utilisées plus haut sont symétriques car les résistances sont des éléments linéaires et réciproques.

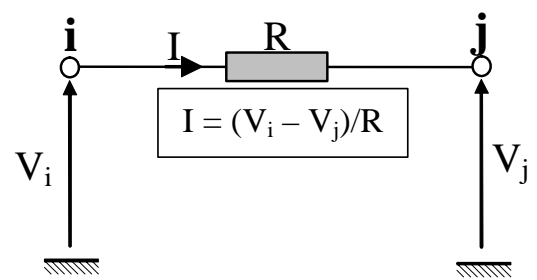

Matrice des conductances associée (symétrique)

$$
[G]=\left[\begin{array}{cc}
1 / R & -1 / R \\
-1 / R & 1 / R
\end{array}\right]
$$

Fig. 4. Elément linéaire réciproque

Un élément linéaire non réciproque conduirait à une matrice non symétrique. Ce cas se rencontre en électricité avec un générateur de courant commandé. Le courant prélevé au nœud i et apporté au nœud j est alors fonction de la tension au nœud $\mathbf{i}$. De ce fait, le nœud $\mathbf{i}$ influe très fortement sur le nœud $\mathbf{j}$, alors que les perturbations au nœud $\mathbf{j}$ n'ont que peu d'influence sur le nœud $\mathbf{i}$.

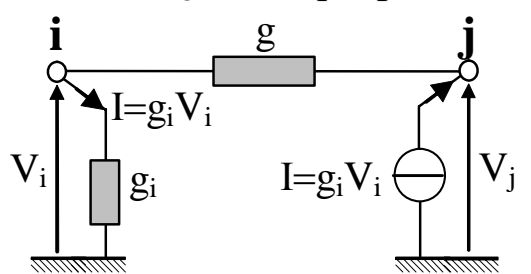

Matrice des conductances associée (non symétrique)

$$
[G]=\left[\begin{array}{cc}
g+g_{i} & -g \\
-g-g_{i} & g
\end{array}\right]
$$

Fig. 5. Elément linéaire non réciproque

Si le réseau comporte des éléments non linéaires comme des diodes, il n’est plus possible d'appliquer la méthode ci-dessus : les différents cas de fonctionnement doivent être traités séparément.

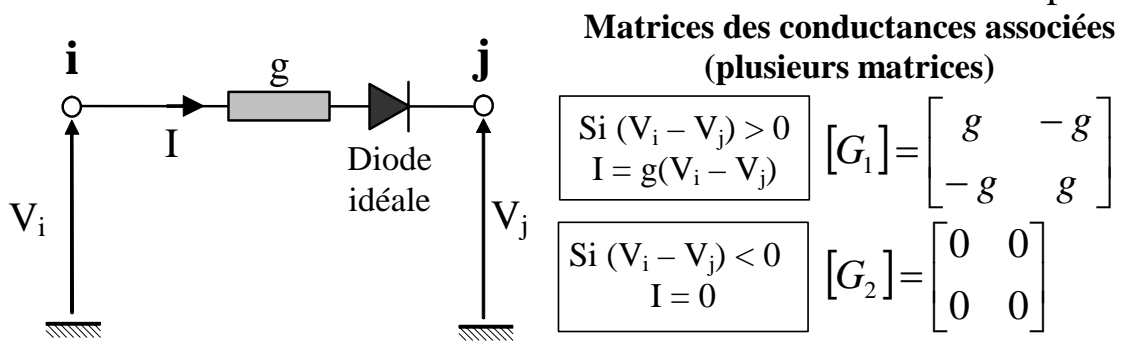

Fig. 6. Elément non linéaire (diode idéale)

\section{Application pratique}

\subsection{Matériel nécessaire}

Pour mettre en pratique les notions précédentes, nous présentons ici une séance de travaux pratiques facile à mettre en œuvre. L'objectif est de déterminer les valeurs d'un ensemble de résistances sans avoir la possibilité d'accéder individuellement à chaque élément. Cette situation se présente concrètement lorsque des résistances ont été assemblées sur un circuit imprimé, et que l'on souhaite effectuer un test ou une vérification des composants en fin d'assemblage. 
Matériel nécessaire à la manipulation :

- une boîte Plexo comportant le réseau de résistances 1/4 W de la série E12 à 10 \% (voir figure 7),

- un multimètre (ohmmètre, voltmètre),

- une alimentation stabilisée,

- une calculatrice dotée de l’inversion matricielle ou un PC avec un logiciel de calcul matriciel.

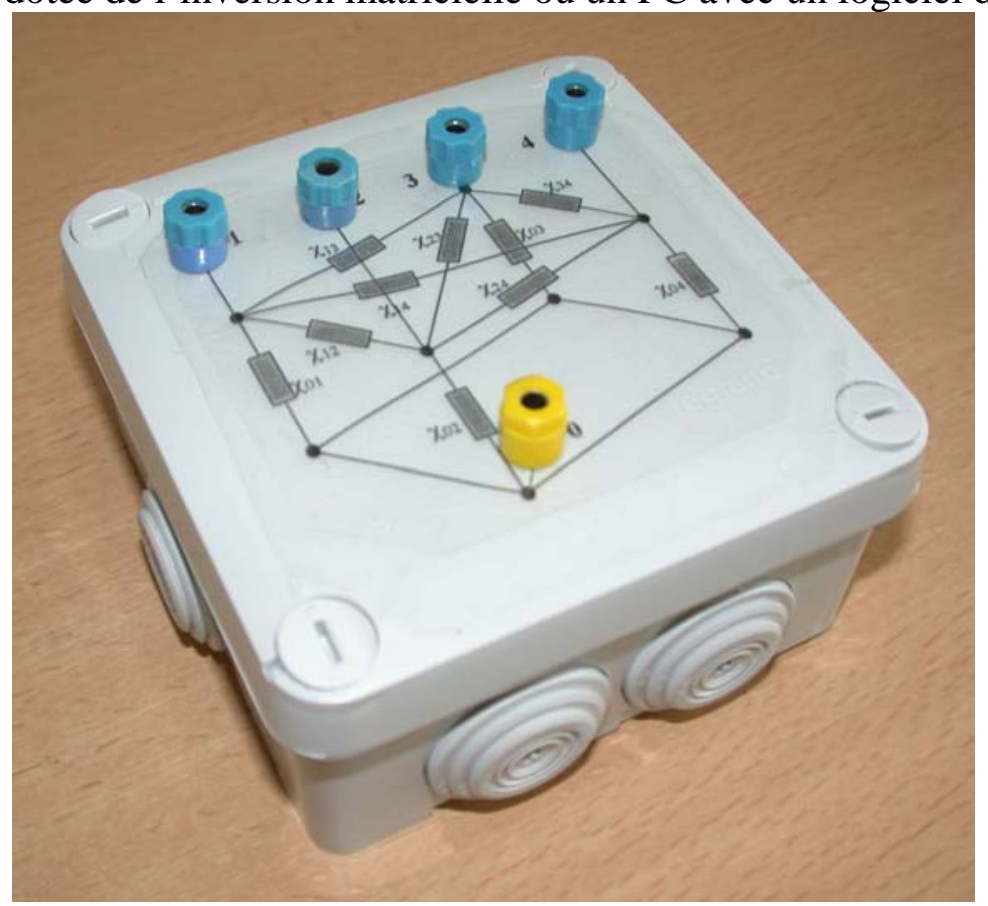

Fig. 7. La boîte Plexo et les bornes d'accès au réseau

Nous disposons dans la boîte d'un réseau de résistances dont les accès sont reliés à des bornes de connexion vers l'extérieur. Nous supposerons que la topologie de ce réseau à quatre nœuds correspond à celle de la figure 8 . L’objectif est de déterminer les valeurs des dix résistances constituant le réseau à partir de simples mesures au niveau des bornes d'accès. Les résistances ne sont pas accessibles individuellement pour effectuer des mesures à l'ohmmètre.

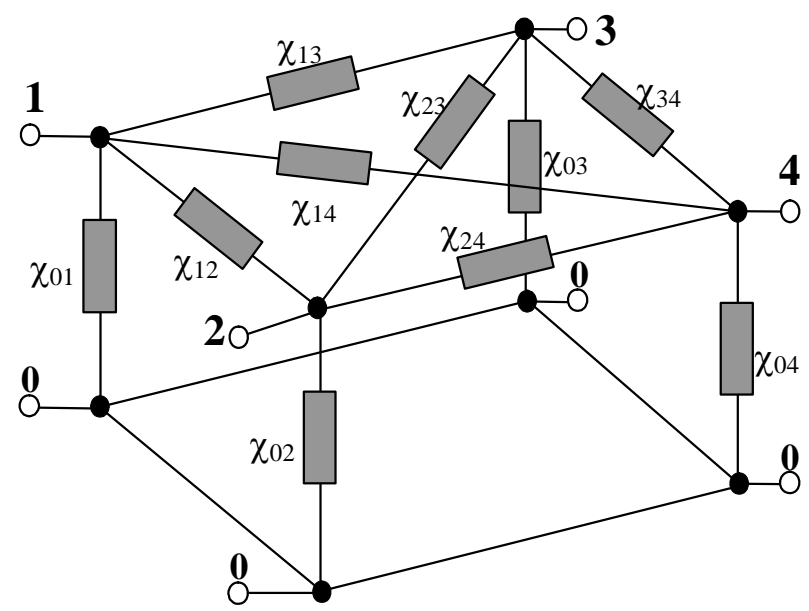

Fig. 8. Le réseau maillé de conductances considéré

La matrice des conductances de ce réseau à 4 nœuds est de dimension 4x4, soit 16 éléments. Mais comme cette matrice est symétrique, il n'y a en réalité que 10 inconnues à déterminer, soit le même nombre que le nombre de résistances à calculer.

\subsection{Les mesures}

\section{Première étape}

On mesure à l'ohmmètre les 4 impédances d'entrée entre les nœuds $(1,2,3,4)$ et le nœud de référence (0). Ceci permet de remplir directement la diagonale de la matrice $[R]$, soit $R_{11}, R_{22}, R_{33}, R_{44}$. Ces quatre impédances correspondent à l’impédance de Thévenin (1857-1926) du réseau (d'entrée ou de sortie, car le réseau est linéaire). 


\section{Deuxième étape}

En imposant une tension fixe à un nœud d'entrée i (ce qui revient à injecter un courant $\mathrm{I}_{\mathrm{i}}$ ), on mesure ensuite la tension aux autres nœuds $\mathbf{j}$. On complète alors la partie non diagonale de la matrice, colonne par colonne, en utilisant le fait que :

$$
R_{j i}=R_{i i} \frac{V_{j}}{V_{i}}
$$

La matrice $[\mathrm{R}]$ est donc remplie de la manière suivante :

$$
[R]=\left[\begin{array}{ccccc}
R_{11} & R_{22} \frac{V_{1}}{V_{2}} & R_{33} \frac{V_{1}}{V_{3}} & R_{44} \frac{V_{1}}{V_{4}} \\
R_{11} \frac{V_{2}}{V_{1}} & R_{22} & R_{33} \frac{V_{2}}{V_{3}} & R_{44} \frac{V_{2}}{V_{4}} \\
R_{11} \frac{V_{3}}{V_{1}} & R_{22} \frac{V_{3}}{V_{2}} & R_{33} & R_{44} \frac{V_{3}}{V_{4}} \\
R_{11} \frac{V_{4}}{V_{1}} & R_{22} \frac{V_{4}}{V_{2}} & R_{33} \frac{V_{4}}{V_{3}} & R_{44}
\end{array}\right]=\left[\begin{array}{lllll}
\mathrm{KKKK} \Omega & \mathrm{KKKK} \Omega & \mathrm{KKKK} \Omega & \mathrm{KKKK} \Omega \\
\mathrm{KKKK} \Omega & \mathrm{KKKK} \Omega & \mathrm{KKKK} \Omega & \mathrm{KKKK} \Omega \\
\mathrm{KKKK} \Omega & \mathrm{KKKK} \Omega & \mathrm{KKKK} \Omega & \mathrm{KKKK} \Omega \\
\mathrm{KKKK} \Omega & \mathrm{KKKK} \Omega & \mathrm{KKKK} \Omega & \mathrm{KKKK} \Omega
\end{array}\right]
$$

Il est possible de contrôler les mesures observées pour la partie supérieure de la matrice en effectuant également les mesures correspondant à la partie inférieure et en vérifiant que la matrice ainsi obtenue est bien symétrique.

\subsection{Inversion matricielle}

Par inversion de la matrice [R], on obtient la matrice [G] qu'il est possible d'interpréter sous la forme du réseau de la figure 8.

$$
[G]=\left[\begin{array}{cccc}
\chi_{01}+\chi_{12}+\chi_{13}+\chi_{14} & -\chi_{12} & -\chi_{13} & -\chi_{14} \\
-\chi_{12} & \chi_{02}+\chi_{12}+\chi_{23}+\chi_{24} & -\chi_{23} & -\chi_{24} \\
-\chi_{13} & -\chi_{23} & \chi_{03}+\chi_{13}+\chi_{23}+\chi_{34} & -\chi_{34} \\
-\chi_{14} & -\chi_{24} & -\chi_{34} & \chi_{04}+\chi_{14}+\chi_{24}+\chi_{34}
\end{array}\right]
$$

\subsection{Détermination des conductances}

Les valeurs des conductances des éléments résistifs composant le réseau sont finalement obtenues à partir de la matrice $[\mathrm{G}]$ :

$\begin{array}{lll}\chi_{01}=G_{11}+G_{12}+G_{13}+G_{14}=\ldots \ldots \ldots \Omega^{-1} & \chi_{12}=-G_{12}=\ldots \ldots \ldots \Omega^{-1} & \chi_{24}=-G_{24}=\ldots \ldots \ldots \Omega^{-1} \\ \chi_{02}=G_{21}+G_{22}+G_{23}+G_{24}=\ldots \ldots \ldots \Omega^{-1} & \chi_{13}=-G_{13}=\ldots \ldots \ldots \Omega^{-1} & \chi_{34}=-G_{34}=\ldots \ldots \ldots \Omega^{-1} \\ \chi_{03}=G_{31}+G_{32}+G_{33}+G_{34}=\ldots \ldots \ldots . \Omega^{-1} & \chi_{14}=-G_{14}=\ldots \ldots \ldots \Omega^{-1} & \\ \chi_{04}=G_{41}+G_{42}+G_{43}+G_{44}=\ldots \ldots \ldots . \Omega^{-1} & \chi_{23}=-G_{23}=\ldots \ldots \ldots \Omega^{-1} & \end{array}$

\begin{tabular}{|c|c|c|c|c|c|}
\hline & Valeurs calculées & Valeurs nominales lues & & Valeurs calculées & Valeurs nominales lues \\
\hline $1 / \chi_{01}$ & ................. & 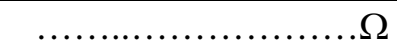 & $1 / \chi_{13}$ & ................. & $\ldots . \Omega$ \\
\hline $1 / \chi_{02}$ &.$\Omega$ &.$\Omega$ & $1 / \chi_{14}$ & $\Omega$ & $\Omega$ \\
\hline $1 / \chi_{03}$ & $\ldots \ldots \ldots \ldots \ldots . \ldots$ & $\ldots . \Omega$ & $1 / \chi_{23}$ & $\ldots \Omega$ &..$\Omega$ \\
\hline $1 / \chi_{04}$ & $\ldots \ldots \ldots \ldots \ldots \ldots$ & $\ldots \ldots \ldots \ldots \ldots \ldots \ldots \ldots \Omega$ & $1 / \chi_{24}$ & $\ldots \ldots \ldots \ldots \ldots \ldots$ & $\ldots \ldots \ldots \ldots \ldots \ldots \ldots \ldots$ \\
\hline $1 / \chi_{12}$ & $\ldots \ldots \ldots \ldots \ldots . \ldots$ & 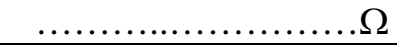 & $1 / \chi_{34}$ & …......... &.$\Omega$ \\
\hline
\end{tabular}

Après ouverture de la boîte (figure 9), on peut effectuer la lecture des valeurs des résistances à l'aide du code des couleurs, et comparer avec les valeurs obtenues par mesure et calcul. 


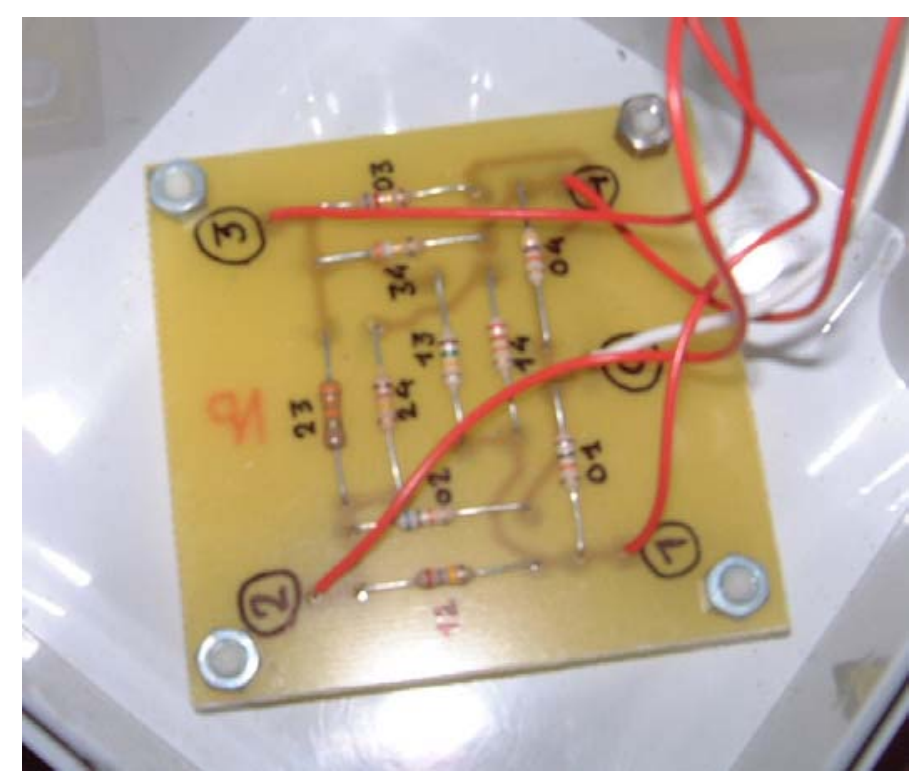

Fig. 9. Le réseau de résistances $1 / 4 \mathrm{~W}$ de la série E12 à $10 \%$ monté sur circuit imprimé à l'intérieur de la boîte Plexo

Les résultats obtenus dans l'exemple traité sont présentés ci-dessous. Pour effectuer les calculs, on peut utiliser un grand nombre de logiciels courants, tels que Maple, MATLAB ou LabView. Nous pouvons nous rendre compte du très bon accord entre les valeurs calculées et les valeurs nominales des composants.

\begin{tabular}{|c|c|}
\hline Valeurs calculées & Valeurs nominales \\
\hline $\mathrm{R}_{01}=9829,19 \Omega$ & $10 \mathrm{k} \Omega \pm 10 \%$ \\
\hline $\mathrm{R}_{02}=68785,62 \Omega$ & $68 \mathrm{k} \Omega \pm 10 \%$ \\
\hline $\mathrm{R}_{03}=803602,21 \Omega$ & $820 \mathrm{k} \Omega \pm 10 \%$ \\
\hline $\mathrm{R}_{04}=47498,65 \Omega$ & $47 \mathrm{k} \Omega \pm 10 \%$ \\
\hline $\mathrm{R}_{12}=268047,86 \Omega$ & $270 \mathrm{k} \Omega \pm 10 \%$ \\
\hline $\mathrm{R}_{13}=149001,36 \Omega$ & $150 \mathrm{k} \Omega \pm 10 \%$ \\
\hline $\mathrm{R}_{14}=22367,91 \Omega$ & $22 \mathrm{k} \Omega \pm 10 \%$ \\
\hline $\mathrm{R}_{23}=33074,66 \Omega$ & $33 \mathrm{k} \Omega \pm 10 \%$ \\
\hline $\mathrm{R}_{24}=119039,07 \Omega$ & $120 \mathrm{k} \Omega \pm 10 \%$ \\
\hline $\mathrm{R}_{34}=385429,17 \Omega$ & $390 \mathrm{k} \Omega \pm 10 \%$ \\
\hline
\end{tabular}

\section{Conclusion}

Cette étude permet une première approche des systèmes linéaires maillés, tout en ne faisant appel qu'à des notions connues.

Elle permet d'utiliser les notions de conductances ou d'admittances, beaucoup moins usuelles à nos étudiants que les notions de résistances et d'impédances. Notons au passage que, sur des systèmes maillés, la notion de conductance apparaît de manière naturelle pour construire la matrice des conductances [G], alors que la notion de résistance est plus compliquée à interpréter au niveau de la matrice des résistances [R].

Cette étude est également l'occasion de revoir la notion d'impédance de Thévenin appliquée à un système déjà complexe et d'apporter les connaissances de base nécessaires à la bonne compréhension du fonctionnement de simulateurs numériques en électricité. Ce dernier sujet dépasserait toutefois le cadre de cet article.

Du point de vue mathématique, cette étude permet l'interprétation physique des éléments d'une matrice et de sa matrice inverse. Elle est donc l'occasion de faire une séance de travaux pratiques transversale entre les enseignements de mathématiques et d’électricité. 


\section{Références}

A. CALVAER, Electricité théorique, Fascicule 2: Analyse des circuits linéaires passifs, Editions Derouaux, B-4000 Liège Belgique, 1978.

P. JOUBERT, Circuits électriques et systèmes : méthodes modernes de calcul, Technique et Documentation Lavoisier, 1986.

R. BOITE, J. NEIRYNCK, Théorie des réseaux de Kirchhoff, Presses polytechniques et universitaires romandes, 1996.

\section{ANNEXE 1}

Session Maple de calcul des résistances

(Maple : logiciel mathématique de calcul formel)

\section{Initialisation des calculs}

> restart: with(linalg):

Warning, new definition for norm

Warning, new definition for trace

Entrée de la matrice $\mathbf{R}$ trouvée d'après les mesures

$\begin{aligned}>\mathrm{R}:=\operatorname{matrix}([ & {[8159.53,3639.61,4370.41,5296.90], } \\ & {[3647.46,31829.38,24579.22,6440.64], } \\ & {[4385.07,24595.92,43863.55,6731.30], } \\ & {[5300.77,6432.00,6714.54,17044.80]]): }\end{aligned}$

\section{Calcul de la matrice $\mathbf{G}$}

$$
\begin{aligned}
& {[3647.46,31829.38,24579.22,6440.64],} \\
& {[4385.07,24595.92,43863.55,6731.30],} \\
& [5300.77,6432.00,6714.54,17044.80]]):
\end{aligned}
$$

$>\mathrm{G}:=\operatorname{evalf}($ inverse(R)) :

\section{Calcul des résistances reliées au nœud de référence}

$>\mathrm{rO1}:=1 /(\mathrm{G}[1,1]+\mathrm{G}[1,2]+\mathrm{G}[1,3]+\mathrm{G}[1,4]) ; \quad \mathrm{rO} 2:=1 /(\mathrm{G}[2,1]+\mathrm{G}[2,2]+\mathrm{G}[2,3]+\mathrm{G}[2,4])$;

$r \odot 3:=1 /(G[3,1]+G[3,2]+G[3,3]+G[3,4]) ; r \odot 4:=1 /(G[4,1]+G[4,2]+G[4,3]+G[4,4])$;

$\mathrm{rO1}:=9829.192838$

r०2:=68785.62334

$\mathrm{r} 03:=803602.2093$

$r \odot 4:=47498.64879$

\section{Calcul des autres résistances}

$>\mathrm{r} 12:=-2 /(\mathrm{G}[1,2]+\mathrm{G}[2,1]) ; \mathrm{r} 13:=-2 /(\mathrm{G}[1,3]+\mathrm{G}[3,1]) ; \mathrm{r} 14:=-2 /(\mathrm{G}[1,4]+\mathrm{G}[4,1])$;

$r 23:=-2 /(\mathrm{G}[2,3]+\mathrm{G}[3,2]) ; \quad r 24:=-2 /(\mathrm{G}[2,4]+\mathrm{G}[4,2]) ; r 34:=-2 /(\mathrm{G}[3,4]+\mathrm{G}[4,3])$;

$$
\begin{aligned}
& \text { r12 }:=268047.8606 \\
& \text { r13 }:=149001.3608 \\
& \text { r14 }:=22367.90988 \\
& \text { r23 }:=33074.66308 \\
& \text { r24 }:=119039.0729 \\
& \text { r34 }:=385429.1650
\end{aligned}
$$




\section{ANNEXE 2}

Session MATLAB de calcul des résistances

(MATLAB : logiciel de simulation pour les sciences de l'ingénieur)

$\%$ Dimension de la matrice

$\mathrm{N}=4$;

\% Entrée de la matrice $\mathrm{R}$ trouvée $\mathrm{d}$ 'après les mesures

$R=\left[\begin{array}{llll}8159.53 & 3639.61 & 4370.415296 .90\end{array}\right.$

$3647.46 \quad 31829.38 \quad 24579.22 \quad 6440.64$

$4385.07 \quad 24595.92 \quad 43863.55 \quad 6731.30$

5300.776432 .006714 .54 17044.80];

$\%$ Calcul de la matrice $\mathrm{G}$

$\mathrm{G}=\operatorname{inv}(\mathrm{R})$;

\% Calcul des résistances reliées au nœud de référence

$r 01=1 /(G(1,1)+G(1,2)+G(1,3)+G(1,4))$;

$r \odot 2=1 /(G(2,1)+G(2,2)+G(2,3)+G(2,4))$;

$r \odot 3=1 /(G(3,1)+G(3,2)+G(3,3)+G(3,4))$;

$\mathrm{r} \odot 4=1 /(\mathrm{G}(4,1)+\mathrm{G}(4,2)+\mathrm{G}(4,3)+\mathrm{G}(4,4))$;

fprintf('Résistances reliées au nœud de référence $\backslash r^{\prime}$ ') ;

fprintf('rO1 = \%E\n', rO1);

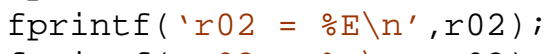

fprintf( 'rO3 = \%E\n', rO3);

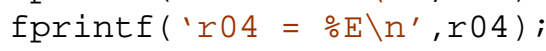

$\%$ Calcul des autres résistances

$\mathrm{r} 12=-2 /(\mathrm{G}(1,2)+\mathrm{G}(2,1))$;

$\mathrm{r} 13=-2 /(\mathrm{G}(1,3)+\mathrm{G}(3,1))$;

$\mathrm{r} 14=-2 /(\mathrm{G}(1,4)+\mathrm{G}(4,1))$;

$\mathrm{r} 23=-2 /(\mathrm{G}(2,3)+\mathrm{G}(3,2))$;

$\mathrm{r} 24=-2 /(\mathrm{G}(2,4)+\mathrm{G}(4,2))$;

$\mathrm{r} 34=-2 /(\mathrm{G}(3,4)+\mathrm{G}(4,3))$;

fprintf('Résistances entre les nœuds $\left.\backslash r^{\prime}\right)$;

fprintf('r12 = \%E\n', r12);

fprintf('r13 = \%E\n', r13);

fprintf('r14 = \%E\n', r14);

fprintf ('r23 = \%E\n', r23);

fprintf('r24 = \%E\n', r24);

fprintf('r34 = \%E\n', r34);

Résistances reliées au nœud de référence

$\mathrm{r} \odot 1=9.829193 \mathrm{E}+003$

$\mathrm{r} \odot 2=6.878562 \mathrm{E}+004$

$\mathrm{r} \odot 3=8.036022 \mathrm{E}+005$

$\mathrm{r} \odot 4=4.749865 \mathrm{E}+004$

Résistances entre les nœuds

$\mathrm{r} 12=2.680479 \mathrm{E}+005$

$\mathrm{r} 13=1.490014 \mathrm{E}+005$

$r 14=2.236791 \mathrm{E}+004$

$r 23=3.307466 \mathrm{E}+004$

$r 24=1.190391 \mathrm{E}+005$

r34 $=3.854292 \mathrm{E}+005$ 


\section{ANNEXE 3}

Session LabView de calcul des résistances

(LabView : logiciel d'informatique industrielle)

\begin{tabular}{|c|c|c|c|c|c|c|c|c|c|c|}
\hline I MA & 3.vi & & & & & & & & & (ax \\
\hline Eichie & Édition Ex & récution Qutils & Parcourir $\mathrm{F}$ & nêtre Aide & & & & & & 周 \\
\hline & 包: & Police de l'appl & cation 12pts & 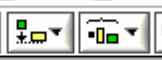 & & & & & & $\frac{19}{197}$ \\
\hline & latrice $R$ & & & & & Matrice G & & & & \\
\hline 5) 0 & 8159,53 & 3639,61 & 4370,41 & 6) 5296,90 & 5) & $1,5686 \mathrm{E}-4$ & $-3,7297 \mathrm{E}-6$ & $5-6,6974 E-6$ & $-4,4691 E-5$ & \\
\hline ㄴ) 0 & 3647,46 & 31829,38 & 단,22 & 드 6440,64 & $\frac{1}{5}$ & $-3,7317 \mathrm{E}-6$ & $5,6899 \mathrm{E}-5$ & $-3,0225 E-5$ & $-8,4039 \mathrm{E}-6$ & \\
\hline & 4385,07 & (5) 24595,92 & 는 43863,55 & 는 6731,30 & & $-6,7253 \mathrm{E}-6$ & $-3,0244 \mathrm{E}-5$ & $4,0813 E-5$ & $-2,5999 \mathrm{E}-6$ & \\
\hline & 5300,77 & 6) 6432,00 & $6 7 \longdiv { 6 7 1 4 , 5 4 }$ & 6) 17044,80 & & $-4,4723 E-5$ & $-8,3973 \mathrm{E}-6$ & $-2,5891 \mathrm{E}-6$ & $7,6763 E-5$ & \\
\hline & R01 & & & R12 & & R14 & & R24 & & \\
\hline & 9829,19 & & 03602,21 & 268047 & & 22367,91 & & 119039,07 & & \\
\hline & R02 & R & & R13 & & R23 & & R34 & & \\
\hline & 68785,6 & & 7498,65 & 149001， & & 33074,66 & & 385429,17 & & \\
\hline 4 & & & & & & & & & & 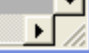 \\
\hline
\end{tabular}

Diagramme de MAT3.vi

Fichier Édition Exécution Quutils Parcourir Fenêtre Aide

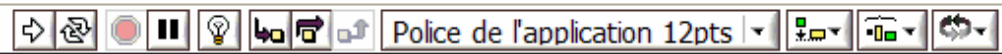

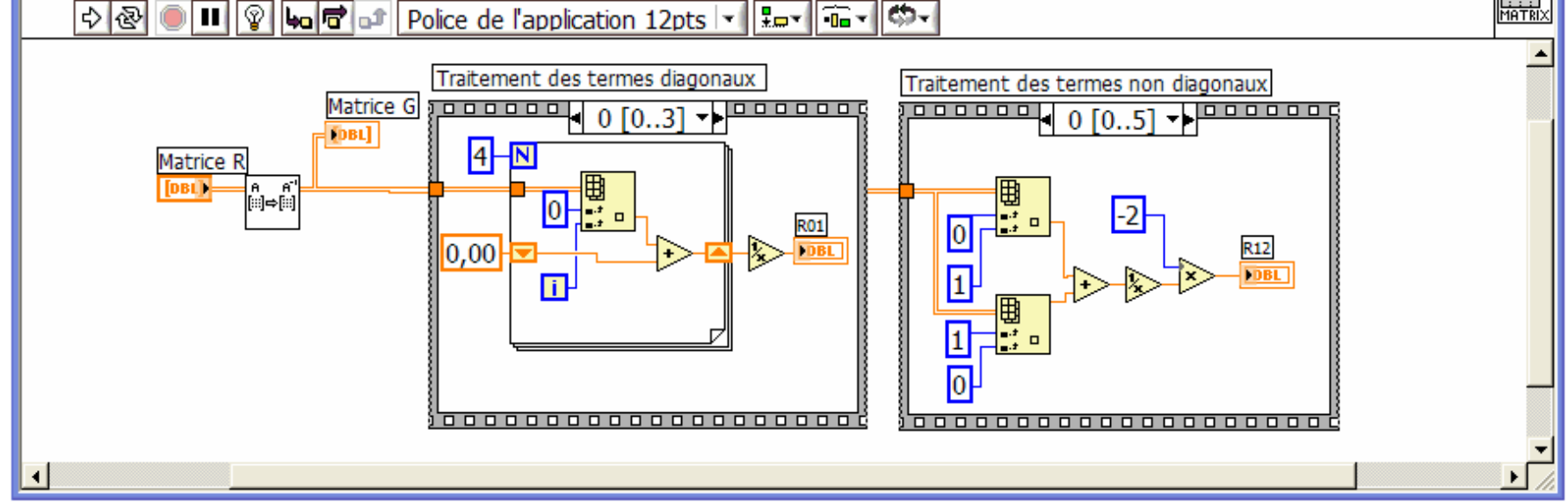

Fig. 10. Exemple de programme LabView permettant de déterminer les valeurs des résistances 

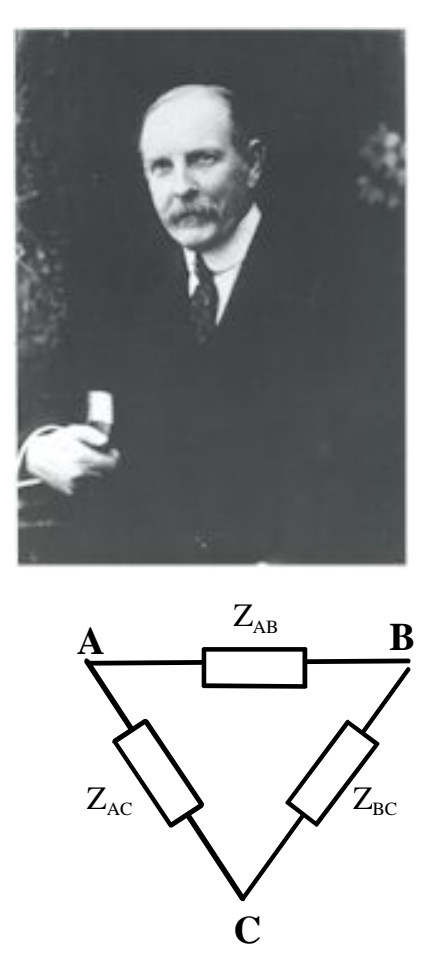

$$
\begin{aligned}
& \mathrm{Z}_{\mathrm{AB}}=\frac{\mathrm{Z}_{\mathrm{A}} \mathrm{Z}_{\mathrm{B}}+\mathrm{Z}_{\mathrm{A}} \mathrm{Z}_{\mathrm{C}}+\mathrm{Z}_{\mathrm{B}} \mathrm{Z}_{\mathrm{C}}}{\mathrm{Z}_{\mathrm{C}}} \mathrm{Z}_{\mathrm{A}}=\frac{\mathrm{Z}_{\mathrm{AB}} \mathrm{Z}_{\mathrm{AC}}}{\mathrm{Z}_{\mathrm{AB}}+\mathrm{Z}_{\mathrm{AC}}+\mathrm{Z}_{\mathrm{BC}}} \\
& \mathrm{Z}_{\mathrm{AC}}=\frac{\mathrm{Z}_{\mathrm{A}} \mathrm{Z}_{\mathrm{B}}+\mathrm{Z}_{\mathrm{A}} \mathrm{Z}_{\mathrm{C}}+\mathrm{Z}_{\mathrm{B}} \mathrm{Z}_{\mathrm{C}}}{\mathrm{Z}_{\mathrm{B}}} \mathrm{Z}_{\mathrm{B}}=\frac{\mathrm{Z}_{\mathrm{AB}} \mathrm{Z}_{\mathrm{BC}}}{\mathrm{Z}_{\mathrm{AB}}+\mathrm{Z}_{\mathrm{AC}}+\mathrm{Z}_{\mathrm{BC}}} \\
& \mathrm{Z}_{\mathrm{BC}}=\frac{\mathrm{Z}_{\mathrm{A}} \mathrm{Z}_{\mathrm{B}}+\mathrm{Z}_{\mathrm{A}} \mathrm{Z}_{\mathrm{C}}+\mathrm{Z}_{\mathrm{B}} \mathrm{Z}_{\mathrm{C}}}{\mathrm{Z}_{\mathrm{A}}} \mathrm{Z}_{\mathrm{C}}=\frac{\mathrm{Z}_{\mathrm{AC}} \mathrm{Z}_{\mathrm{BC}}}{\mathrm{Z}_{\mathrm{AB}}+\mathrm{Z}_{\mathrm{AC}}+\mathrm{Z}_{\mathrm{BC}}}
\end{aligned}
$$



Arthur Edwin KENNELLY, Électrotechnicien américain (Coloba, près de Bombay, 1861 - Boston 1939). Une des plus importantes contributions qu'il a apportées à l'électrotechnique est constituée par un mémoire sur l'« impédance », présenté en 1893 devant l'American Institute of Electrical Engineers et où, pour la première fois, on trouve l'application des quantités complexes à la loi d'Ohm en courant alternatif. En 1902, Kennelly a exposé sa théorie bien connue de l'influence de l'ionisation solaire dans l'atmosphère sur les transmissions radioélectriques à longue distance, théorie qui a été vérifiée expérimentalement depuis lors, et qui est à l'origine de la conception de la couche ionisée de réflexion appelée « couche de Kennelly-Heaviside ».

Fig. 12. Transposition de Kennelly

Biographies des auteurs :

\section{Patrick LAGONOTTE}

I.U.T. de Poitiers, Département HSE, 8, rue Archimède, 79000 Niort

Laboratoire d’Etudes Thermiques, UMR CNRS n6608, ENSMA, BP 40109, 86961 Futuroscope Cedex, France.

Tél : 05-49-49-81-23

Fax : 05-49-49-81-01

E-mail : lagonotte@let.ensma.fr

Ancien élève de l'École Normale Supérieure de Cachan, professeur agrégé de Génie Électrique en 1984, Docteur de l’Institut National Polytechnique de Grenoble en 1987. Il est Maître de Conférences à l’Université de Poitiers depuis 1988 où il enseigne l'électrotechnique et est l'auteur d'un ouvrage sur les installations électriques. Il effectue ses recherches au Laboratoire d'Etudes Thermiques de l'ENSMA de Poitiers. Ses principaux domaines de recherche sont la modélisation des systèmes, la thermique des machines électriques, le refroidissement des semi-conducteurs de puissance, les équations de diffusion et de propagation, la modélisation des systèmes d’ordre non entier ou d’ordre infini, la caractérisation des systèmes électrochimiques.

\section{Yves EICHENLAUB}

I.U.T. de Poitiers, Département GEII, 6 allée Jean Monnet, 86010 Poitiers Cedex

Laboratoire de Mathématiques et Applications, UMR CNRS n6086, BP 30179, 86962 Futuroscope Cedex, France.

Tél : 05-49-45-34-91

Fax : 05-49-45-38-80

E-mail : yves.eichenlaub@univ-poitiers.fr

Ancien élève de l’École Normale Supérieure, il est agrégé de mathématiques et docteur de l’Université Bordeaux 1 spécialité Mathématiques Pures. Maître de Conférences à l’Université de Poitiers depuis 1997, il y enseigne les mathématiques et est spécialisé dans le domaine des algorithmes en algèbre et en théorie des nombres. 